ARTICLE

Received 23 Apr 2015 | Accepted 18 May 2015 | Published 1 Jul 2015

DOI: $10.1038 /$ ncomms 8550

\title{
Bivalent separation into univalents precedes age-related meiosis I errors in oocytes
}

\author{
Yogo Sakakibara', Shu Hashimoto², Yoshiharu Nakaoka², Anna Kouznetsova ${ }^{3}$, Christer Höög ${ }^{3}$ \\ \& Tomoya S. Kitajima ${ }^{1}$
}

The frequency of chromosome segregation errors during meiosis I (MI) in oocytes increases with age. The two-hit model suggests that errors are caused by the combination of a first hit that creates susceptible crossover configurations and a second hit comprising an age-related reduction in chromosome cohesion. This model predicts an age-related increase in univalents, but direct evidence of this phenomenon as a major cause of segregation errors has been lacking. Here, we provide the first live analysis of single chromosomes undergoing segregation errors during $\mathrm{MI}$ in the oocytes of naturally aged mice. Chromosome tracking reveals that $80 \%$ of the errors are preceded by bivalent separation into univalents. The set of the univalents is biased towards balanced and unbalanced predivision of sister chromatids during MI. Moreover, we find univalents predisposed to predivision in human oocytes. This study defines premature bivalent separation into univalents as the primary defect responsible for age-related aneuploidy.

\footnotetext{
${ }^{1}$ Laboratory for Chromosome Segregation, RIKEN Center for Developmental Biology, Kobe 650-0047, Japan. ${ }^{2}$ IVF Namba Clinic, Osaka 550-0015, Japan. ${ }^{3}$ Department of Cell and Molecular Biology, Karolinska Institutet, Stockholm 171 77, Sweden. Correspondence and requests for materials should be addressed to T.S.K. (email: tkitajima@cdb.riken.jp).
} 
neuploidy is a leading cause of miscarriage and congenital diseases such as Down syndrome. Most aneuploid chromosomes originate from segregation errors during meiosis I (MI) in oocytes, and the frequency of the errors increases with age ${ }^{1-4}$. The two-hit model suggests that segregation errors during MI are caused by the combination of a first hit that creates susceptible crossover configurations during the foetal stage and a second hit comprising an age-related reduction in chromosome cohesion during the dictyate stage ${ }^{1,5-8}$. One prediction of this model is the existence of an age-related increase in univalents. Consistent with this prediction, $S m c 1 b$ knockout oocytes, which have a reduced amount of cohesin, exhibit an age-related increase in univalents ${ }^{9}$. However, direct evidence of univalents as a major cause of segregation errors in the oocytes of naturally aged animals has been lacking ${ }^{10-15}$. Although an age-related increase in univalents in the oocytes of naturally aged mice was first suggested over four decades ago ${ }^{16}$, it was argued that most, if not all, of the univalents observed in that study were artefacts of chromosome spread ${ }^{10-12}$. Recent studies found univalents in a very small fraction of the oocytes of naturally aged mice ${ }^{13,14}$, which seemed unlikely to account for the majority of chromosome segregation errors during MI. These and other studies found weakly associated bivalents in a significant fraction of the oocytes of naturally aged mice ${ }^{13-15}$, but these bivalents undergo normal segregation during $\mathrm{MI}^{13}$. Instead, available evidence from naturally aged mice led to the proposal that an age-related reduction in cohesion between sister kinetochores (KTs) ${ }^{14,15}$ and defects in KT-microtubule interaction $^{17}$ are leading causes of segregation errors during MI. Thus, the actual cause of segregation errors during MI in the oocytes of naturally aged animals remains unclear.

Here, we provide direct evidence that premature bivalent separation into univalents is the major cause of chromosome segregation errors during MI. By using high-resolution and a
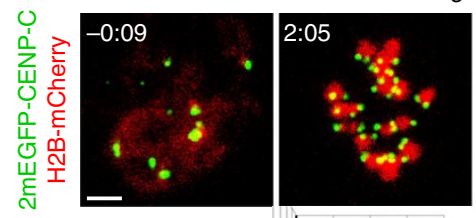

$5: 13$
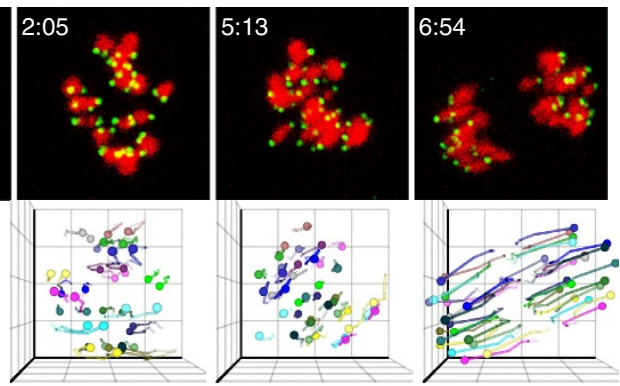

Aged oocyte with MI error
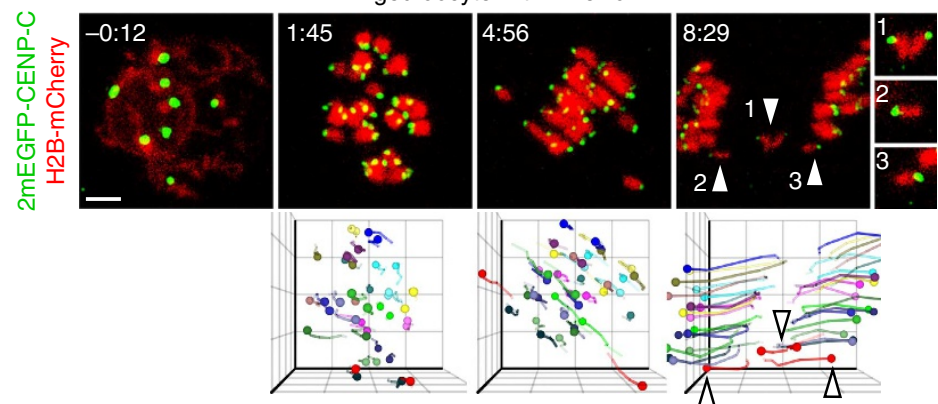

b

Anaphase KT tracks
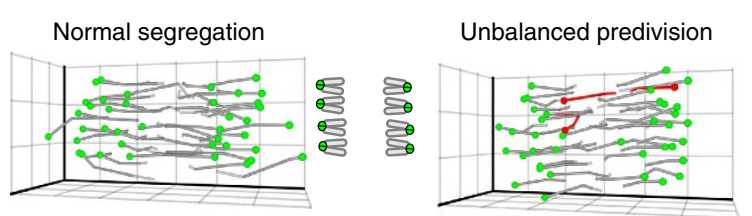

Balanced predivision
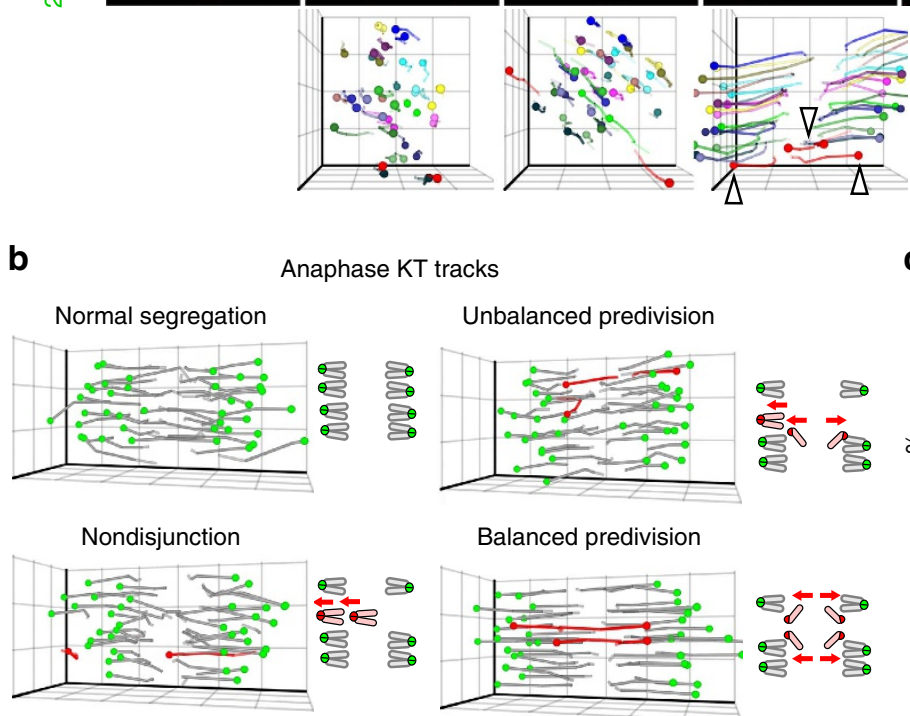

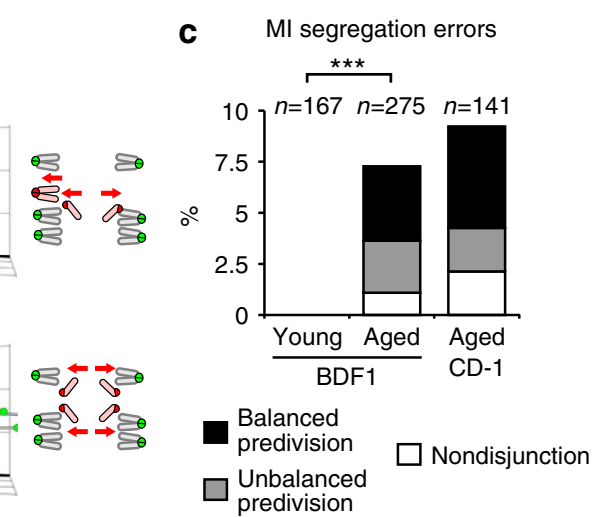

Figure 1 | Live imaging of $\mathbf{M l}$ errors in aged mouse oocytes. (a) Live imaging and tracking of KTs. Maximum z-projection images of 2mEGFP-CENP-C (KTs, green) and H2B-mCherry (chromosomes, red) in the oocytes of young (2 months old) and aged (16 months old) BDF1 mice. KT signals are peak-enhanced and background-subtracted. The 3D plots show KT positions (spheres) and their tracks (lines). Same colours were used for homologous KTs. The aged oocyte underwent balanced predivision of sister chromatids (arrowheads and insets). Time after NEBD (h:mm). Scale bar, $5 \mu$ m. See also Supplementary Movie 2. (b) Distinct types of segregation errors during MI. Red spheres and lines indicate KTs that have segregated abnormally. See also Supplementary Fig. 1. (c) Balanced and unbalanced predivision are predominant over nondisjunction. The rates of segregation errors during MI in the oocytes from young BDF1, aged BDF1, and aged CD-1 (11 months old) mice. Fisher's exact test was performed. ${ }^{\star \star \star} P<0.001$. 
high-throughput live imaging microscopy and complete threedimensional (3D) tracking of KTs in the oocytes of naturally aged mice, we show that the vast majority of chromosome segregation errors are preceded by bivalent hyperstretching and subsequent separation into univalents. The univalents are strongly biased towards predivision of sister chromatids. We provide direct observation of balanced predivision of sister chromatids during MI, which was hypothesized two decades ago ${ }^{5}$, as a major type of age-related chromosome segregation errors. Consistent with observations in mouse oocytes, human oocytes exhibit univalents that are predisposed to predivision of sister chromatids during MI.

\section{Results}

Direct observation of age-related MI errors. There are potentially three distinct types of segregation errors during MI: nondisjunction of homologous chromosomes (4:0 segregation), unbalanced predivision of sister chromatids (3:1 segregation) and balanced predivision of sister chromatids (2:2 segregation) (Supplementary Fig. 1). Balanced predivision ${ }^{5}$, in which both homologous chromosomes undergo sister chromatid segregation during MI, has not yet been directly demonstrated because it is indistinguishable from premature sister chromatid separation during meiosis II (MII) by conventional approaches using fixed metaphase II oocytes. To directly observe chromosome

a
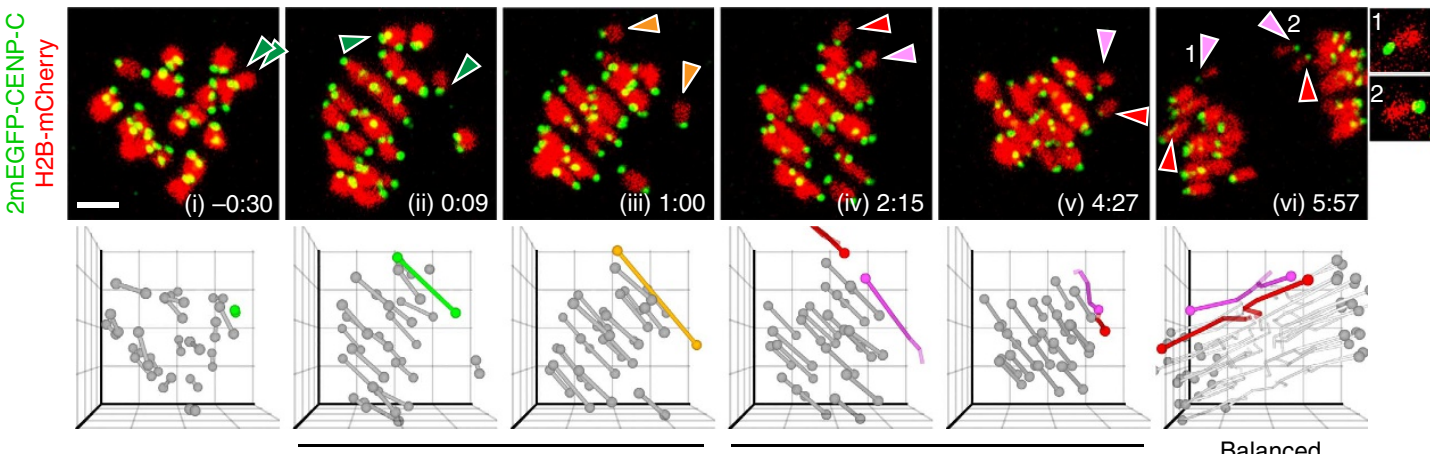

Independent movement

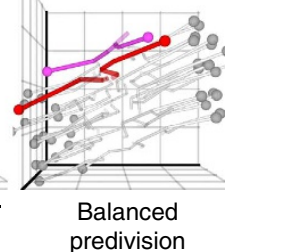

b

Distance from equator

C
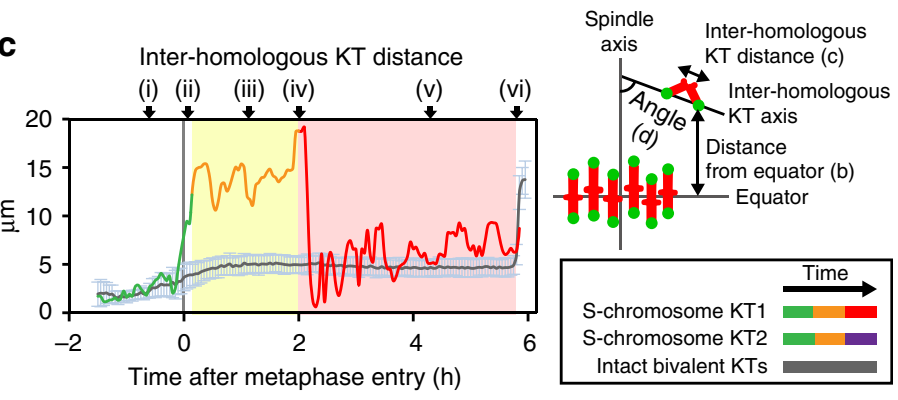

d

e

KT speed

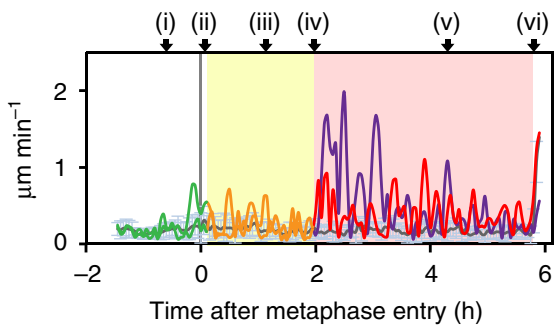

f

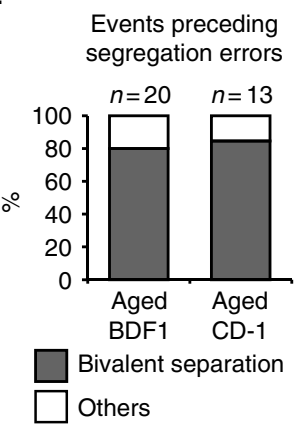

Figure 2 | Bivalent separation prior to chromosome segregation errors. (a) Bivalent hyperstretching and separation before segregation errors. Maximum z-projection images of aged (16 months old) BDF1 oocytes expressing 2mEGFP-CENP-C (KTs, green) and H2B-mCherry (chromosomes, red). KT signals are peak-enhanced and background-subtracted. Arrowheads indicate S-chromosome KTs. The 3D plots show KT positions (spheres) and the connection between homologous KTs (lines). In (i) - (ii), the S-chromosome is coloured in green. In (iii), the hyperstretched S-chromosome is coloured in orange. In (iv) - (vi), the positions (spheres) and trajectories (lines) of the S-chromosome KTs exhibiting independent movements are coloured in red and purple. Intact bivalents are coloured in grey. The insets show the predivision of sister chromatids. Note that the spindle rotated before (vi). Time after metaphase entry (h:mm). Scale bar, $5 \mu \mathrm{m}$. See also Supplementary Movie 4. (b-e) Characteristic S-chromosome dynamics suggests bivalent hyperstretching followed by premature separation. KT distance from the spindle equator (b), inter-homologous KT distance (c), angle between inter-homologous KT axis and the spindle axis (d) and KT speed (e). The data for S-chromosomes are coloured as in $\mathbf{a}$. The data for bivalents are coloured in grey. Error bars, s.d. (f) Bivalent separation precedes the majority of segregation errors during MI. The KT trajectories that underwent segregation errors in oocytes from aged BDF1 (16 months old) and CD-1 (11 months old) mice were analysed. 


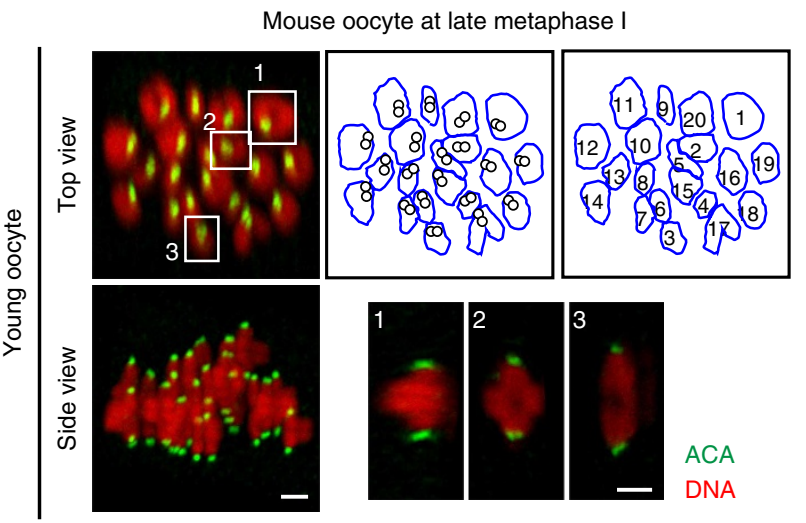

b
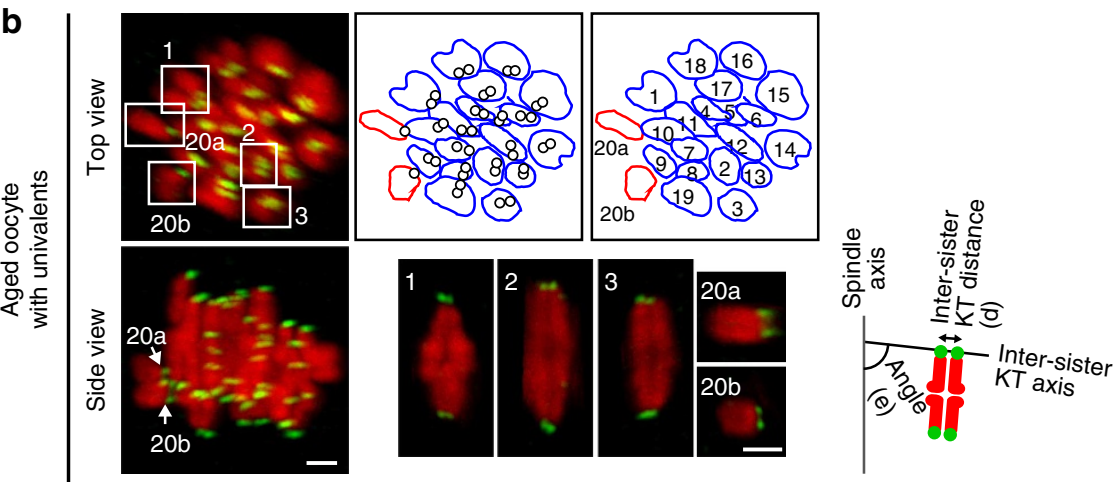

C

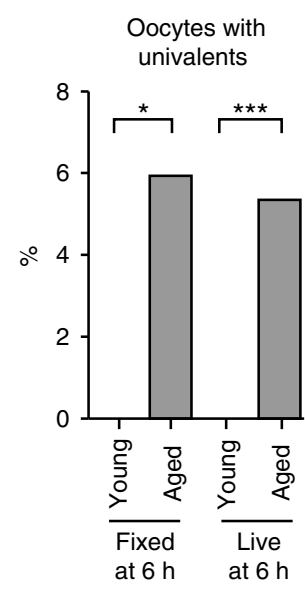

d

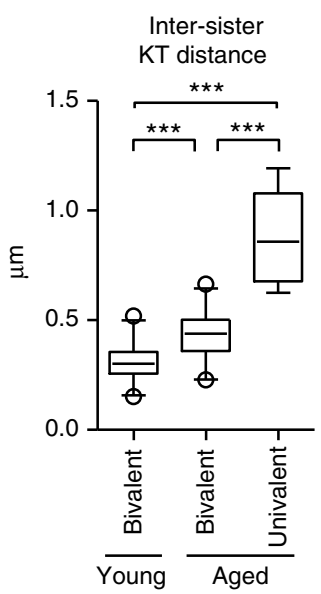

e

Inter-sister KT axis angle

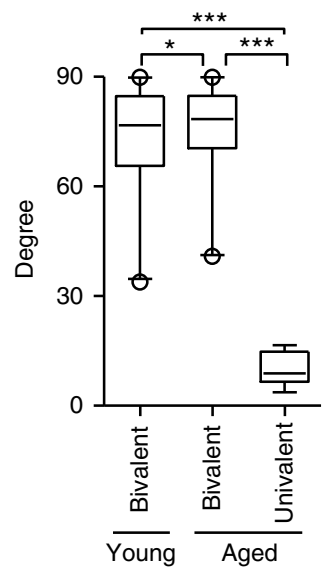

Figure 3 | Age-related increase in univalents in mouse oocytes. (a,b) Univalents in fixed aged oocytes. Oocytes from young (2 months old (a)) and aged (16 months old (b)) BDF1 mice were cultured and fixed at $6 \mathrm{~h}$ after NEBD. The oocytes were stained for KTs (ACA, green) and DNA (Hoechst 33342, red). KT signals are peak-enhanced and background-subtracted. All homologous KT positions were determined in 3D and the number of chromosomes was counted. Projection views from the top and side of the spindle are shown with signal interpolation in z. The outlines of chromosomes (bivalents, blue; univalents, red) and the positions of homologous KTs (circles) are shown. Representative bivalents and univalents are magnified. Scale bars, $2 \mu \mathrm{m}$. See also Supplementary Movie 6. (c) The rate of oocytes with univalents. In fixed oocytes, separated chromosome units that have one of the homologous KTs and an inter-homologous KT axis with a tilt angle of $>25^{\circ}$ with respect to the spindle axis were categorized as univalents $(n=106,118)$. The same criteria was used for live oocytes at $6 \mathrm{~h}$ after $\operatorname{NEBD}(n=180,289)$. Note that the rates of univalents presented here is underestimated because this criteria excludes univalents that are indistinguishable from hyperstretched bivalents at a single time point. The rate of univalents determined by an analysis of multiple time points in live aged oocytes was $9.5 \%$ (Supplementary Table 1). Fisher's exact test was performed. ${ }^{\star} P=0.015,{ }^{\star \star \star} P=0.0004$. (d,e) Univalents are predisposed to predivision. The inter-sister KT distance (c) and the angle between inter-sister KT axis and the spindle axis $(\mathbf{d})$ were measured $(n=160,152,8)$. Boxes show the 25th-75th percentiles and whiskers show 1-99 percentiles. Two-tailed, unpaired Student's $t$-test was performed. ${ }^{\star} P<0.05$, ${ }^{\star \star \star} P<0.001$.

segregation errors, we established a high-resolution and highthroughput imaging of KTs and chromosomes in live mouse oocytes (Fig. 1a and Supplementary Movie 1) ${ }^{18}$. We generated four-dimensional data sets throughout MI for 275 'aged' oocytes obtained from 16-month-old BDF1 mice and for 167 'young' oocytes obtained from 2-month-old mice (Supplementary Table 1). The data sets allowed us to robustly track all KTs in 3D from prometaphase to anaphase I (Fig. 1a, Supplementary Movie 2) ${ }^{18}$. Manual assessment of all KT trajectories during anaphase I (Fig. 1b) indicated that 20/275 aged oocytes (7.3\%) 
a

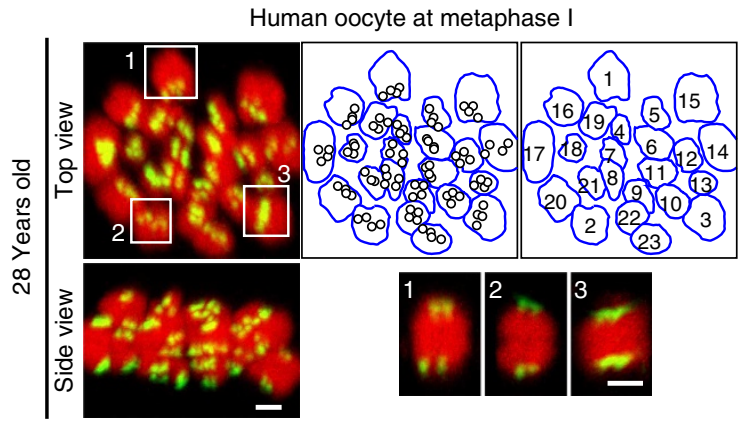

b
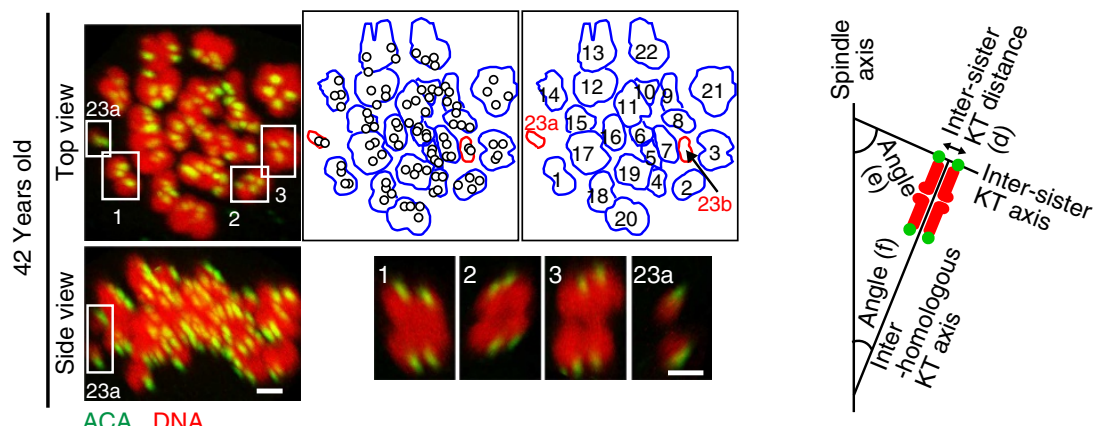

C

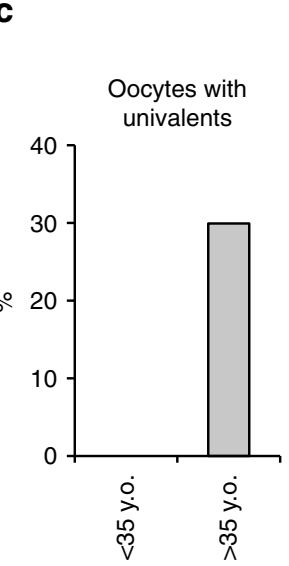

d

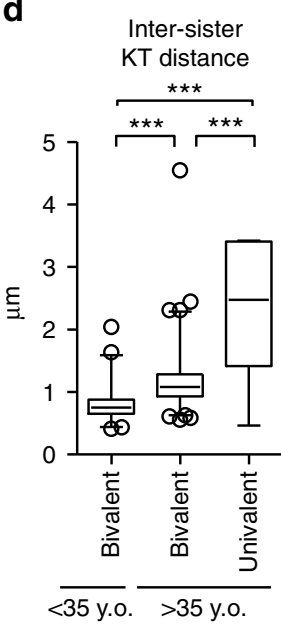

e

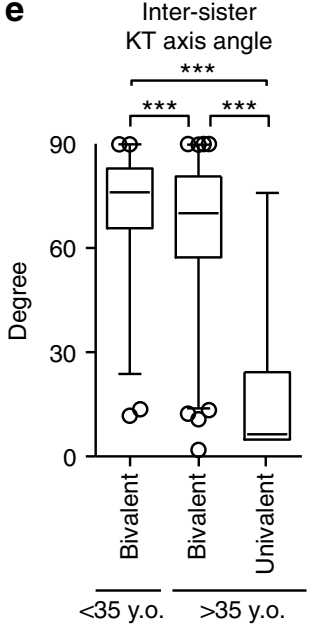

f

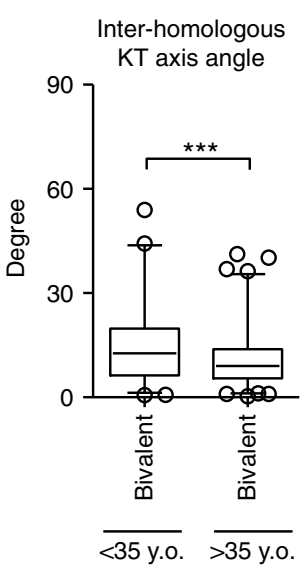

Figure 4 | Univalents predisposed to predivision in human oocytes. (a,b) Univalents in human oocytes. Oocytes were stained for KTs (ACA, green) and DNA (Hoechst 33342, red). Oocytes from 28-year old (a) and 42-year-old (b) patients are shown. KT signals are peak-enhanced and backgroundsubtracted. All sister and homologous KT positions were determined in 3D and the number of chromosomes was counted. Projection views from the top and side of the spindle are shown with signal interpolation in $z$. The outlines of chromosomes (bivalents, blue; univalents, red) and the positions of KTs (circles) are shown. Representative bivalents and univalents are magnified. Scale bars, $2 \mu \mathrm{m}$. See also Supplementary Movies 7 and 8 . (c) The rate of oocytes with univalents. Separated chromosome units that have one of the homologous KTs and an inter-homologous KT axis with a tilt angle of $>25^{\circ}$ with respect to the spindle axis were categorized as univalents $(n=6,10)$. (d,e) Univalents are predisposed to predivision. The inter-sister KT distance (d) and the angle between the inter-sister KT axis and the spindle axis (e) were measured $(n=276,454,6)$. (f) Homologous chromosome biorientation. The angle between the inter-homologous KT axis and the spindle axis was measured $(n=276,454)$. Boxes show the 25 th-75th percentiles and whiskers show 1-99 percentiles. Two-tailed, unpaired Student's $t$-test was performed. ${ }^{\star \star \star} P<0.001$. y.o., years old.

underwent segregation errors during $\mathrm{MI}$, whereas no young oocytes did (0/167; Fig. 1c and Supplementary Table 1). This KT tracking analysis revealed that balanced predivision of sister chromatids $(9 / 20,45 \%)$ and unbalanced predivision of sister chromatids $(7 / 20,35 \%)$ are predominant over nondisjunction of homologous chromosomes (4/20, 20\%) (Fig. 1c). Similar results were obtained for oocytes from aged CD-1 mice $(7 / 13,54 \%$ balanced predivision; 3/13, 23\% unbalanced predivision; and $3 / 13,23 \%$ nondisjunction) (Fig. 1c). Counting the number of KTs in aged BDF1 oocytes fixed at metaphase II suggested that live imaging had no adverse effect on the rates of unbalanced predivision and nondisjunction (Supplementary Fig. 2 and Supplementary Movie 3). These results indicate that segregation errors during MI are strongly biased towards predivision of sister chromatids, including balanced predivision.

Bivalent separation into univalents precedes MI errors. Strikingly, analysis of the KT trajectories of the chromosomes later associated with segregation errors suggested premature bivalent separation (Supplementary Movie 4). Hereafter, we refer to these chromosomes as 'S-chromosomes'. Before metaphase I, 


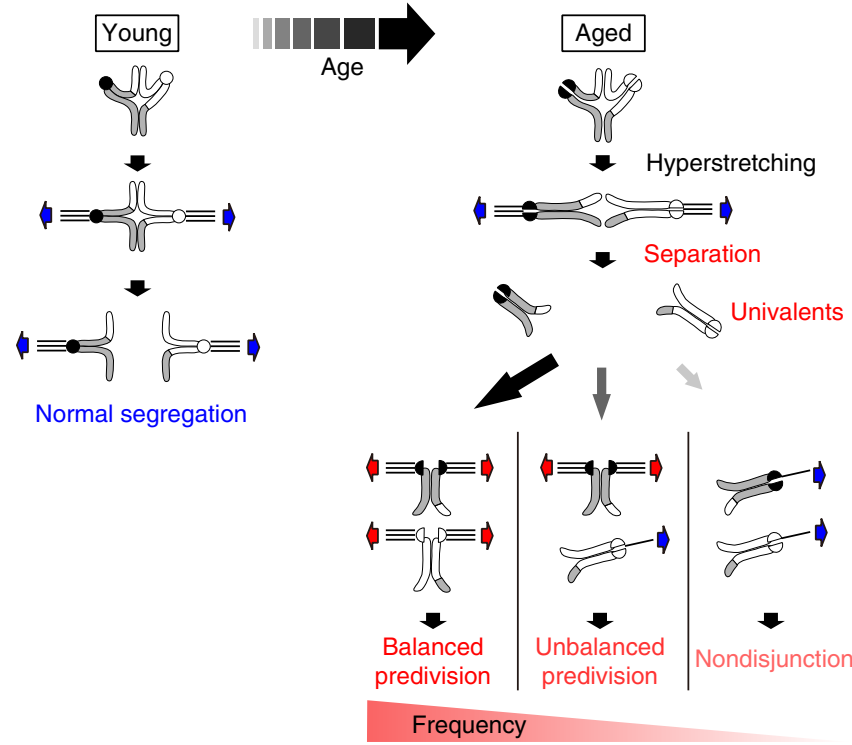

Figure 5 | Premature bivalent separation into univalents precedes age-related chromosome segregation errors during MI. Bivalents with weakened cohesion in aged oocytes undergo hyperstretching and separation into univalents by microtubule-mediated bipolar spindle forces. This bipolar forces in turn biorient the sister KTs of the univalents. The univalents are therefore strongly biased towards predivision of sister chromatids, including balanced predivision. After balanced predivision during $\mathrm{MI}$, the chromatids undergo random segregation during MII.

an S-chromosome appeared as a normal bivalent with a pair of homologous KTs (Fig. 2a(i)). Upon entry into metaphase I, the S-chromosome bivalent stretched with a similar timing to that of the other bivalents (Fig. 2a-c(ii)). This stretching resulted in an extremely long distance between the homologous KTs (interhomologous KT distance; Fig. 2a,c(iii)). Although no detectable $\mathrm{H} 2 \mathrm{~B}$-mCherry signal was observed in the middle of the hyperstretched bivalent (Fig. 2a(iii)), it was possible that the homologous chromosomes were still functionally connected because the line connecting the homologous KTs (inter-homologous KT axis) was stably oriented along the spindle axis for a maximum of 233 min (Fig. 2b,d and Supplementary Fig. 3). This observation of hyperstretched bivalents is consistent with a previous report ${ }^{13}$. Thereafter, however, we found that the homologous KTs of the S-chromosome suddenly began to oscillate independently between the spindle poles at an increased speed (Fig. 2a-e(iv) and Supplementary Movie 4). The characteristic profiles of this oscillation were indistinguishable from those of univalents generated due to a defect in synaptonemal complex formation in the oocytes of young $S y c p 3$ knockout mice (Supplementary Fig. 4 and Supplementary Movie 5) ${ }^{19}$. We therefore functionally define these apparently separated S-chromosome units exhibiting independent oscillation as univalents. Importantly, of the 20 chromosomes that exhibited segregation errors during MI in aged BDF1 oocytes, 16 (80\%) showed bivalent hyperstretching followed by separation into univalents (Fig. 2f, Supplementary Fig. 3 and Supplementary Table 1). The same phenomenon preceded $85 \%$ (11/13) of segregation errors during MI in oocytes from aged CD-1 mice (Fig. $2 \mathrm{f}$ and Supplementary Fig. 5). These results suggest that premature bivalent separation into univalents precedes the majority of chromosome segregation errors during MI in the oocytes of aged mice.

Age-related increase in univalents in mouse oocytes. To confirm the age-related increase in univalents, we fixed and immunostained oocytes at late metaphase I without chromosome spreading. Determination of all KT positions in $3 \mathrm{D}$ revealed that a significant fraction of aged oocytes had univalents, whereas no young oocytes did (Fig. 3a-c and Supplementary Movie 6). The rate of univalents remained consistent between fixed and live oocytes (Fig. 3c). The sister KTs of these univalents were frequently bioriented and aligned on the metaphase plate (Fig. 3b,d,e), which was consistent with the observation of univalents that later exhibited sister chromatid predivision in live aged oocytes (Supplementary Fig. 6). These results confirm the age-related increase in univalents as the major cause of segregation errors during MI in mouse oocytes.

Univalents in human oocytes. To investigate whether univalents are observed in human oocytes, we fixed and immunostained oocytes at MI from patients of different ages without chromosome spreading. Determination of all KT positions in 3D (Fig. 4a and Supplementary Movie 7) revealed that among 16 oocytes carrying a metaphase plate, three oocytes from relatively old patients contained univalents (Fig. 4b,c and Supplementary Movie 8). The sister KTs of the univalents were frequently bioriented and aligned on the metaphase plate (Fig. 4b,d,e), which suggests a predisposition to sister chromatid predivision during MI. On bivalents, the inter-sister KT distance was significantly greater in oocytes from older patients (Fig. 4d), as observed in mouse oocytes (Fig. $3 \mathrm{~d})^{14,15}$. This age-related reduction in sister $\mathrm{KT}$ cohesion had a significant but relatively small impact on the capacity of bivalents to biorient the homologous KTs (Fig. 4f) and to monoorient the sister KTs (Fig. 4e), whereas univalent formation strongly biased towards biorientation of the sister KTs (Fig. 4e). These results suggest that univalent formation, presumably through premature bivalent separation as observed in mouse oocytes, is a critical event for chromosome segregation errors during $\mathrm{MI}$ in human oocytes.

\section{Discussion}

It has long been speculated that an age-related increase in univalents might be a leading cause of chromosome segregation errors in oocytes ${ }^{5-7,9,16,19}$. In 1968, Henderson and Edwards ${ }^{16}$ observed an age-related increase in univalents in chromosome spread samples of the oocytes from naturally aged mice. Recent studies detected univalents in the oocytes from naturally aged mice without chromosome spreading ${ }^{13,14}$, although the sample size in these studies was insufficient to show statistical significance and the rate of the detected univalents did not match the rate of chromosome segregation errors. Instead, these and other studies observed a significant age-related increase in weakly associated bivalents and sister $\mathrm{KTs}^{13-15}$, raising the possibility that these defects are a leading cause of chromosome segregation errors.

In the present study, our systematic analyses of the dynamics and structures of single chromosomes in the oocytes of naturally aged mice provided direct evidence that premature bivalent separation into univalents is the major cause of age-related chromosome segregation errors during MI (Fig. 5). Consistent with this finding, we observed univalents predisposed to segregation errors in human oocytes, although this observation should be interpreted cautiously because oocytes that remained immature after hormonal stimulation were used in this study. In mouse oocytes, a small fraction of segregation errors were not preceded by premature bivalent separation $(4 / 20,20 \%$ in BDF1; $2 / 13,15 \%$ in CD-1; Supplementary Table 1). Future studies are needed to determine whether these errors are attributed to an age-related loss of sister KT cohesion on intact bivalents ${ }^{14,15}$ 
or other defects that facilitate incorrect KT-microtubule attachments ${ }^{17}$.

Our observation of premature bivalent separation into univalents is consistent with a prediction of the two-hit model $^{1,5-8}$. Our findings suggest that the functional connection between the homologous chromosomes of bivalents subjected to both hits is resolved by microtubule-mediated forces for bivalent stretching, which thus results in production of univalents (Fig. 5). The possibility that a leak of separase activity contributed to the bivalent separation cannot be excluded. The sister KTs of the univalents are strongly biased towards bipolar microtubule attachment, possibly for two reasons. First, monopolar microtubule attachments to the sister KTs fail to be stabilized due to a loss of tension between homologous KTs, whereas bipolar attachments can be stabilized by the tension between sister $\mathrm{KTs}^{19,20}$. Second, an age-related loss of sister KT cohesion $^{14,15}$ may disrupt MI-specific sister KT geometry that is known to be essential for univalents, but largely dispensable for bivalents, to make monopolar microtubule attachments to the sister $\mathrm{KTs}^{21,22}$.

Consequently, the set of the univalents frequently results in balanced and unbalanced predivision of sister chromatids during MI (Fig. 5). Unbalanced predivision is consistent with the aneuploidy observed in human metaphase II oocytes $5,7,23-27$. Furthermore, balanced predivision, which was demonstrated to be the most predominant type of segregation errors during $\mathrm{MI}$ in mouse oocytes in this study, may reconcile the available human data. DNA polymorphism analyses of human trisomies have shown that most aneuploid chromosomes are caused by segregation errors during $\mathrm{MI}^{1-4}$, whereas genome-based DNA analyses of human oocytes reveal that chromosome number abnormalities are more frequent after MII than after $\mathrm{MI}^{25,28}$. This apparent discrepancy can be reconciled by balanced predivision during MI, which results in the normal number of chromatids after MI but a predisposition to an abnormal number after MII. Taking these findings together, we suggest that premature bivalent separation into univalents is a predominant cause of age-related segregation errors during MI in human oocytes. This study provides a basis for the development of strategies to prevent age-related production of aneuploid eggs, such as the introduction of an artificial tie that could physically secure homologous chromosomes until the onset of anaphase I in maturing oocytes.

\section{Methods \\ Oocyte culture and microinjection of mRNA. Two- or 16-month-old BDF1, 11-month-old CD-1 and 2-month-old Sycp $3^{-/-}$(in C57BL/6 background) female mice were primed with an injection of pregnant mare's serum gonadotropin (Serotoropin, ASKA Pharmaceutical). Ovaries were excised and oocytes at the germinal vesicle stage were collected. The collected oocytes were cultured in M2 medium supplemented with $200 \mathrm{nM} 3$-isobutyl-1-metyl-xanthine (IBMX, Sigma). In vitro-transcribed mRNAs (1.2 pl of $650 \mathrm{ng}^{-1} \mathrm{l}^{-1} 2 \mathrm{mEGFP}-\mathrm{CENP}-\mathrm{C}$ and $0.6 \mathrm{pl}$ of $350 \mathrm{ng} \mathrm{l}^{-1} \mathrm{H} 2 \mathrm{~B}$-mCherry) were microinjected into oocytes. The microinjected oocytes were cultured for $2.5-3.0 \mathrm{~h}$ and then released to undergo meiotic maturation through the removal of IBMX from the culture. Oocytes that expressed a moderate level of H2B-mCherry at the germinal vesicle stage were used for further analysis. All experiments involving mice were approved (AH26-04) and carried out under the guidelines of RIKEN CDB.}

Time-lapse imaging data acquisition. Time-lapse imaging was performed as previously described ${ }^{18}$ with some modifications. Briefly, a Zeiss LSM710 confocal microscope equipped with a $40 \times$ C-Apochromat $1.2 \mathrm{NA}$ water immersion objective lens (Carl Zeiss) was controlled by a multi-position autofocus macro ${ }^{29}$, which allowed us to record a maximum 32 oocytes in one experiment. Seventeen confocal $z$-sections (every $1.5 \mu \mathrm{m}$ ) of $256 \times 256$ pixel $x y$ images covering a total volume of $30.4 \times 30.4 \times 25.5 \mu \mathrm{m}$ were acquired at 3 -min intervals for at least $12 \mathrm{~h}$ after inducing a resumption of meiosis. The KT signals were peak-enhanced and background-subtracted as previously described ${ }^{18}$.
KT tracking. KT tracking was performed as previously described ${ }^{18}$. The distance between homologous KTs (inter-homologous KT distance) at 15 min before the onset of anaphase I was defined as the final inter-homologous KT distance. The inter-homologous KT distance at each time point was normalized to the final inter-homologous KT distance. Metaphase entry was defined as the time when $>50 \%$ of bivalents maintained a minimum inter-homologous KT distance that exceeded $70 \%$ for three consecutive time points. Once the inter-homologous KT distance of a bivalent has exceeded $12 \mu \mathrm{m}$, this bivalent was defined as hyperstretched. Once the homologous KTs of a bivalent initiated independent oscillation, this bivalent was defined as separated into univalents.

The centre of the spindle was defined as the centre of mass of all KT positions. The orientation of the spindle axis was defined as the average orientation of the inter-homologous KT axes of bivalents showing an inter-homologous KT distance $>70 \%$. The KT positions of univalents were eliminated from the calculations of the centre and axis of the spindle. The plane orthogonal to the spindle axis and passing through the spindle centre was defined as the spindle equator. The spindle equator at the time of metaphase entry was used for all time points before the metaphase entry.

To measure the oscillation amplitude, oscillation frequency and KT speed, the KT tracks were fitted with a cubic smoothing spline. The initiation of independent movements of separated chromosome units was defined as the point at which the KT began to exhibit consecutive anti-poleward movement over three time points, followed by a displacement of over $10 \mu \mathrm{m}$ within $15 \mathrm{~min}$. The time point when a KT began to exhibit consecutive poleward or anti-poleward movement over three time points was defined as the turning point. The oscillation amplitude was defined as the KT displacement along the spindle axis from a turning point to the next. The oscillation frequency was defined as the number of turning points after a KT displacement over $10 \mu \mathrm{m}$.

Immunostaining of mouse oocytes. Oocytes that underwent nuclear envelope breakdown (NEBD) within $60 \mathrm{~min}$ after the induction of meiotic resumption were collected either $6 \mathrm{~h}$ after NEBD (late metaphase I) or 12-14 h after NEBD (metaphase II). The oocytes were fixed with $1.6 \%$ paraformaldehyde in $100 \mathrm{mM}$ PIPES pH 7.0, $1 \mathrm{mM} \mathrm{MgCl}_{2}$ and $0.1 \%$ Triton X-100 for $30 \mathrm{~min}$ at room temperature. The fixed oocytes were washed three times with PBT $(0.1 \%$ Triton $\mathrm{X}-100$ in PBS) and then blocked with $3 \% \mathrm{BSA}-\mathrm{PBT}$ at $4{ }^{\circ} \mathrm{C}$ overnight. The oocytes were then incubated with human anti-centromere antibodies (ACA, Antibodies Incorporated, 15-234) at 1:100 in 3\% BSA-PBT at $4{ }^{\circ} \mathrm{C}$ overnight. The oocytes were subsequently washed three times with 3\% BSA-PBT and incubated with an Alexa Fluor 488 goat anti-human antibody (Molecular Probes, A11013) at 1:500 in 3\% BSA-PBT at room temperature for $4 \mathrm{~h}$ in the dark. DNA was counterstained with $40 \mu \mathrm{g} \mathrm{ml}^{-1}$ Hoechst 33342. The oocytes were finally washed again and transferred to $0.01 \%$ BSA-PBS for imaging with a Zeiss LSM780 confocal microscope.

Confocal $z$-sections (every $0.2 \mu \mathrm{m}$ ) of $512 \times 512$ pixel $x y$ images covering the total volume of the spindle were acquired. The images were reconstructed into $3 \mathrm{D}$ with Imaris software (Bitplane)

Human oocyte experiments. Human oocyte experiments were approved by institutional human research ethics committees at RIKEN (KOBE-IRB-13-22) and IVF Namba Clinic (2014-1), registered in Japan Society of Obstetrics and Gynecology (registry number 132), and carried out under these guidelines. Immature oocytes that were not used for fertility treatment were donated after patients received a full explanation of the experiments and provided signed informed consent.

Controlled ovarian stimulation was performed by using standard protocols with modifications according to the patients' medical history. Oocytes were picked up by transvaginal aspiration with an 18 gauge single lumen needle (Smiths Medical) $36 \mathrm{~h}$ after human chorionic gonadotropin injection. All mature oocytes were inseminated by intracytoplasmic sperm injection for fertility treatment. Oocytes that were still immature at $42 \mathrm{~h}$ after human chorionic gonadotropin injection were donated for this study. The immature oocytes were fixed in $2 \%$ paraformaldehyde solution at $37^{\circ} \mathrm{C}$ for $30 \mathrm{~min}$. Immunostaining was performed by the same procedure that was used for mouse oocytes except for primary antibody incubation for two overnights. The oocytes were imaged with a Zeiss LSM780 confocal microscope equipped with a $63 \times \mathrm{C}$-Apochromat $1.2 \mathrm{NA}$ water immersion objective lens. To remove oocytes that were degenerate or otherwise aberrant, we selected oocytes that exhibit a metaphase plate with or without misaligned chromosomes for high-resolution imaging. Confocal $z$-sections (every $0.2 \mu \mathrm{m}$ ) of $512 \times 512$ pixel $x y$ images covering the total volume of the spindle were acquired. The images were reconstructed into 3D with Imaris software.

\section{References}

1. Hassold, T. \& Sherman, S. Down syndrome: genetic recombination and the origin of the extra chromosome 21. Clin. Genet. 57, 95-100 (2000)

2. Chiang, T., Schultz, R. M. \& Lampson, M. A. Meiotic origins of maternal age-related aneuploidy. Biol. Reprod. 86, 1-7 (2012).

3. Nagaoka, S. I., Hassold, T. J. \& Hunt, P. A. Human aneuploidy: mechanisms and new insights into an age-old problem. Nat. Rev. Genet. 13, 493-504 (2012). 
4. Jones, K. T. \& Lane, S. I. Molecular causes of aneuploidy in mammalian eggs. Development 140, 3719-3730 (2013).

5. Angell, R. R. Predivision in human oocytes at meiosis I: a mechanism for trisomy formation in man. Hum. Genet. 86, 383-387 (1991).

6. Lamb, N. E. et al. Susceptible chiasmate configurations of chromosome 21 predispose to non-disjunction in both maternal meiosis I and meiosis II. Nat. Genet. 14, 400-405 (1996).

7. Angell, R. First-meiotic-division nondisjunction in human oocytes. Am. J. Hum. Genet. 61, 23-32 (1997).

8. Jessberger, R. Age-related aneuploidy through cohesion exhaustion. EMBO Rep. 13, 539-546 (2012).

9. Hodges, C. A., Revenkova, E., Jessberger, R., Hassold, T. J. \& Hunt, P. A. SMClbeta-deficient female mice provide evidence that cohesins are a missing link in age-related nondisjunction. Nat. Genet. 37, 1351-1355 (2005).

10. Polani, P. E. \& Jagiello, G. M. Chiasmata, meiotic univalents, and age in relation to aneuploid imbalance in mice. Cytogenet. Cell Genet. 16, 505-529 (1976).

11. Speed, R. M. The effects of ageing on the meiotic chromosomes of male and female mice. Chromosoma 64, 241-254 (1977).

12. Sugawara, S. \& Mikamo, K. Maternal ageing and nondisjunction: a comparative study of two chromosomal techniques on the formation of univalents in first meiotic metaphase oocytes of the mouse. Chromosoma 93, 321-325 (1986).

13. Yun, Y., Lane, S. I. \& Jones, K. T. Premature dyad separation in meiosis II is the major segregation error with maternal age in mouse oocytes. Development 141, 199-208 (2014)

14. Chiang, T., Duncan, F. E., Schindler, K., Schultz, R. M. \& Lampson, M. A. Evidence that weakened centromere cohesion is a leading cause of age-related aneuploidy in oocytes. Curr. Biol. 20, 1522-1528 (2010).

15. Lister, L. M. et al. Age-related meiotic segregation errors in mammalian oocytes are preceded by depletion of cohesin and Sgo2. Curr. Biol. 20, 1511-1521 (2010).

16. Henderson, S. \& Edwards, R. Chiasma frequency and maternal age in mammals. Nature 218, 22-28 (1968).

17. Shomper, M., Lappa, C. \& FitzHarris, G. Kinetochore microtubule establishment is defective in oocytes from aged mice. Cell Cycle 13, 1171-1179 (2014).

18. Kitajima, T. S., Ohsugi, M. \& Ellenberg, J. Complete kinetochore tracking reveals error-prone homologous chromosome biorientation in mammalian oocytes. Cell 146, 568-581 (2011).

19. Kouznetsova, A., Lister, L., Nordenskjöld, M., Herbert, M. \& Höög, C. Bi-orientation of achiasmatic chromosomes in meiosis I oocytes contributes to aneuploidy in mice. Nat. Genet. 39, 966-968 (2007).

20. LeMaire-Adkins, R., Radke, K. \& Hunt, P. A. Lack of checkpoint control at the metaphase/anaphase transition: a mechanism of meiotic nondisjunction in mammalian females. J. Cell Biol. 139, 1611-1619 (1997).

21. Sakuno, T., Tada, K. \& Watanabe, Y. Kinetochore geometry defined by cohesion within the centromere. Nature 458, 852-858 (2009).

22. Kim, J. et al. Meikin is a conserved regulator of meiosis-I-specific kinetochore function. Nature 517, 466-471 (2015).

23. Vialard, F. et al. Evidence of a high proportion of premature unbalanced separation of sister chromatids in the first polar bodies of women of advanced age. Hum. Reprod. 21, 1172-1178 (2006).
24. Gabriel, A. S. et al. Array comparative genomic hybridisation on first polar bodies suggests that non-disjunction is not the predominant mechanism leading to aneuploidy in humans. J. Med. Genet. 48, 433-437 (2011).

25. Handyside, A. H. et al. Multiple meiotic errors caused by predivision of chromatids in women of advanced maternal age undergoing in vitro fertilisation. Eur. J. Hum. Genet. 20, 742-747 (2012).

26. Forman, E. J. et al. Embryos whose polar bodies contain isolated reciprocal chromosome aneuploidy are almost always euploid. Hum. Reprod. 28, 502-508 (2013).

27. Pellestor, F., Andréo, B., Arnal, F., Humeau, C. \& Demaille, J. Mechanisms of non-disjunction in human female meiosis: the co-existence of two modes of malsegregation evidenced by the karyotyping of 1397 in-vitro unfertilized oocytes. Hum. Reprod. 17, 2134-2145 (2002).

28. Fragouli, E. et al. The cytogenetics of polar bodies: insights into female meiosis and the diagnosis of aneuploidy. Mol. Hum. Reprod. 17, 286-295 (2011).

29. Rabut, G. \& Ellenberg, J. Automatic real-time three-dimensional cell tracking by fluorescence microscopy. J. Microsc. 216, 131-137 (2004).

\section{Acknowledgements}

We thank Dr J. Ellenberg for a macro for automated confocal microscopy, the CDB imaging and animal facilities for supports. This work was supported by a RIKEN Special Postdoctoral Fellowship to Y.S., grants from IVF Namba Clinic to S.H. and Y.N., grants from the Swedish Cancer Society, the Swedish Research Council and the Karolinska Institutet to C.H. and A.K., and grants from Nakajima Foundation, Uehara Memorial Foundation and RIKEN CDB to T.S.K.

\section{Author contributions}

Y.S. performed the experiments and data analysis. S.H. and Y.N. prepared human oocyte samples. A.K. and C.H. assisted Sycp3 knockout experiments. T.S.K. designed the study and wrote the manuscript with input from all authors.

\section{Additional information}

Supplementary Information accompanies this paper at http://www.nature.com/ naturecommunications

Competing financial interests: The authors declare no competing financial interests

Reprints and permission information is available online at http://npg.nature.com/ reprintsandpermissions/

How to cite this article: Sakakibara, Y. et al. Bivalent separation into univalents precedes age-related meiosis I errors in oocytes. Nat. Commun. 6:7550 doi: 10.1038/ncomms8550 (2015).

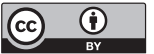

This work is licensed under a Creative Commons Attribution 4.0 International License. The images or other third party material in this article are included in the article's Creative Commons license, unless indicated otherwise in the credit line; if the material is not included under the Creative Commons license, users will need to obtain permission from the license holder to reproduce the material. To view a copy of this license, visit http://creativecommons.org/licenses/by/4.0/ 